\title{
Transtornos do espectro do autismo e educação inclusiva: análise de atitudes sociais de professores e alunos frente à inclusão
}

\author{
Maria Cláudia Brito*
}

\section{Resumo}

O objetivo deste estudo foi descrever as atitudes sociais de professores e alunos que compóem o ambiente escolar de crianças com transtornos do espectro do autismo, acerca da inclusáo. Participaram 73 educadores e 116 alunos, de quatro escolas públicas, sendo duas escolas sem experiência de educação inclusiva e duas escolas com crianças com transtornos do espectro do autismo, de $5^{\circ}$. ano, gênero masculino, 10 anos de idade. Foram utilizadas com as duas crianças com transtornos do espectro do autismo provas de avaliação de linguagem e de comportamentos. Para verificar as atitudes sociais dos professores, empregou-se a Escala Likert de Atitudes Sociais em relação à Inclusão e dos alunos a Escala Infantil de Atitudes Sociais. Os resultados mostraram que não houve diferenças nas atitudes sociais dos professores, e que as atitudes sociais expressas pelos colegas da criança com transtornos do espectro do autismo de quadro clínico menos severo foram mais positivas do que as dos outros três grupos.

Palavras-chave: Transtornos do espectro do autismo; Transtorno global do desenvolvimento; Atitudes sociais; Educação Especial.

* Pós-doutora em Educação pela Universidade Estadual Paulista Júlio de Mesquita Filho, Marília, São Paulo, Brasil. 


\section{Autism spectrum disorders and inclusive education: analysis of social attitudes of teachers and students toward inclusion}

\section{Abstract}

The objective of this study was to describe the social attitudes of teachers and students that are constituent of the school environment of children with autism spectrum disorders, about inclusion. 73 teachers and 116 students from four public schools, two schools with no experience of inclusive education and two schools with children with autism spectrum disorders took part in the study. The two children with autism spectrum disorders were 10 years old boys attending 5 th grade. Tests of language and behavior assessment were used with the two children with autism spectrum disorders. In order to measure the social attitudes of the teachers, the Likert Scale of Social Attitudes toward Inclusion was used. For the measure of student's social attitudes, the Child Scale of Social Attitudes was used. The results showed that there were no differences among the scores of four groups of teachers. The social attitudes of the peers of the child with autism spectrum disorders with a less severe clinical symptom were more positive than the social attitudes of other three groups.

Keywords: Autism spectrum disorders; Developmental disorder; Social attitudes; Special Education.

\section{Introdução}

As crianças com transtornos do espectro do autismo (TEA) estáo entre as mais rejeitadas por seus pares da mesma faixa etária e são percebidas mais negativamente do que crianças com desenvolvimento típico (CAMPBELL; MARINO, 2009; CAMPBELL, 2006; SWAIM; MORGAN, 2001) e com outros transtornos do desenvolvimento (SWAIM; MORGAN, 2001).

A frequente presença de uma aparência física normal associada a características bastante específicas não denota motivo explícito para a ocorrência dos comportamentos incomuns, o que comumente gera, por parte de outras pessoas, atitudes sociais negativas em relação a essas crianças com transtornos do espectro do autismo (CAMPBELL, 2008; CHAMBRES et al., 2008; CAMPBELL, 2006). Portanto, é evidente a necessidade de pesquisas e intervençóes que abordem as atitudes sociais e suas contribuiçóes para tornar a escola um contexto social mais acolhedor e adequado para os alunos com transtornos do espectro do autismo e toda comunidade discente.

Conforme Omote et al. (2005), as atitudes sociais da comunidade escolar frente à inclusão permitem compreender as condiçôes de acolhimento dos alunos, sendo essenciais para a viabilização de alteraçôes no ambiente social das escolas. A avaliação das atitudes sociais de professores em relação à inclusão auxilia na compreensão das condutas que eles adotam em suas salas de aula (ALAHBABI, 2009; HSIEN, 2007; OMOTE et al., 2005) e do compromisso que têm em relação ao 
desenvolvimento e à aceitação de crianças com deficiências como parte da comunidade escolar (HSIEN, 2007). Nessa perspectiva, o objetivo do presente estudo foi o de descrever as atitudes sociais de professores e alunos que compóem o ambiente escolar de alunos com transtornos do espectro do autismo, acerca da inclusão.

\section{Método}

Este estudo foi autorizado mediante assinatura do Termo de Consentimento Livre e Esclarecido, elaborado para fins específicos desta pesquisa, segundo a Resolução no. 196/96 e teve aprovação do Comitê de Ética em Pesquisa da instituição sob o protocolo número 0151/2011. Para retribuir a colaboração dos participantes deste estudo, os pesquisadores ofereceram assessoria acerca do desenvolvimento de linguagem de crianças com transtornos do espectro do autismo e sobre como favorecer as interaçôes sociais dessas crianças em contexto escolar. Tal procedimento foi realizado em grupo com as professoras das crianças participantes da pesquisa e outros professores da escola.

\section{Participantes}

Esta pesquisa contou com 189 participantes, sendo 73 professores e 116 alunos de quatro escolas públicas de Ensino Fundamental de uma cidade de médio porte do interior do Estado de São Paulo. Duas destas escolas tinham inseridas em salas de ensino regular uma criança com transtornos do espectro do autismo e as outras duas escolas não possuíam experiência de educaçáo inclusiva, sendo seus alunos utilizados como grupo controle. Assim, os participantes foram distribuídos em quatro grupos: Grupo 1 - 47 educadores de duas Escolas Estaduais de Ensino Fundamental, que tinham inserido em classe comum um aluno com transtornos do espectro do autismo; Grupo 2 - 26 educadores de duas Escolas Estaduais de Ensino Fundamental sem experiência de educaçáo inclusiva; Grupo 3 - duas crianças com transtornos do espectro do autismo, do gênero masculino, com 10 anos de idade, cujos diagnósticos foram realizados por psiquiatra, segundo critérios específicos e 51 crianças sem deficiências, que estavam em suas classes, totalizando 53 crianças; e Grupo 4 - 63 crianças das escolas sem experiência de educação inclusiva, também matriculadas em $5^{\circ}$ ano.

As crianças com transtornos do espectro do autismo são aqui denominadas participantes focais 1 e 2 (P1 e P2), uma vez que a pesquisa tem como foco principal investigar aspectos referente ao meio social em ambiente escolar dessas crianças. Embora aquelas com transtornos do espectro do autismo tivessem diagnósticos estabelecidos, não havia nas escolas prontuários que permitissem a descrição detalhada de suas manifestaçóes clínicas. Portanto, incluiu-se neste estudo a avaliação do perfil comportamental dos P1 e P2 por meio da aplicação das provas de Pragmática (FERNANDES, 2004) e de Vocabulário (BEFI-LOPES, 2004) e da Escala de Avaliação de Traços Autísticos (ASSUMPÇÃO, et al., 2008), conforme descrito nos Procedimentos. Essa avaliação era necessária, pois há evidências de que as atitudes sociais e a aceitação ou rejeição em relação à inclusão de crianças com transtornos do espectro do autismo podem ser influenciadas por variáveis como o tipo e a severidade das alteraçôes apresentadas. 
Foi possível verificar que o P1 apresentou alterações clínicas menos severas. Observa-se que os P1 e P2 demonstraram comunicar-se preferencialmente pelo meio gestual, seguido do meio verbal, sendo que as iniciativas do P1 foram principalmente expressas por funçóes comunicativas mais interativas e as do P2 por funçôes menos interativas. Ambas as crianças apresentaram utilização de vocábulos usuais, mas com desempenho aquém do esperado para faixa etária e nível de escolaridade delas. $\mathrm{Na}$ Escala de Avaliação de Traços Autísticos (ASSUMPÇÃO, et al., 2008), os P1 e P2 obtiveram escore acima da nota de corte $(23,0)$, o que caracteriza a presença de transtorno global do desenvolvimento, sendo que o P2 demonstrou expressiva pontuação nos itens referentes a problemas de comportamento associados à agressividade.

\section{Procedimentos}

A coleta de dados iniciou-se por meio de entrevista individual com as máes dos P1 e P2, para o preenchimento da ficha informativa elaborada especificamente para esta pesquisa, apenas com o objetivo de caracterizar os participantes do estudo. Foi também solicitado que as professoras dos P1 e P2 preenchessem a ficha informativa no que se referem à caracterização delas próprias.

A Prova de Pragmática consiste na identificação e na descrição do perfil comunicativo do indivíduo e visa analisar os aspectos funcionais da comunicação. Para sua aplicaçáo foram levados alguns objetos de interesse dos P1 e P2, selecionados com base em indicaçóes das mães e professoras dos mesmos, como livros, lápis de cor, papel e alguns objetos lúdicos. Foi entáo realizada uma filmagem de 30 minutos de cada participante em interação com a pesquisadora. Os dados registrados em vídeo foram sintetizados em protocolos individuais que identificaram número de atos comunicativos, funçôes comunicativas expressas e meios comunicativos utilizados, segundo os critérios sugeridos por Fernandes (2004).

Na Prova de Verificação do Vocabulário, realizada também em uma sessão individual com cada criança, a pesquisadora sentou-se à mesa de frente para criança, com pranchas de 118 figuras divididas em nove campos conceituais, entáo foi questionando sobre as mesmas. A sessão foi também registrada por uma filmadora posicionada sobre um tripé, para posterior transcrição das respostas, registro no Protocolo de Registro de Resposta e análise dos dados (BEFI-LOPES, 2004).

A Escala de Avaliação de Traços Autísticos - ATA (ASSUMPÇÃO, et al., 2008) foi preenchida conjuntamente com a máe de cada criança, questionando se a criança apresentava os comportamentos mencionados na escala. $\mathrm{Na}$ análise dos dados, foi calculado o escore individual dos P1 e P2, sendo ponto de corte o valor 23, acima do qual, o escore é indicativo de que a criança apresenta um distúrbio do espectro autístico.

Para mensurar as atitudes sociais dos 73 educadores em relação à inclusão, a Escala Likert de Atitudes Sociais em Relação à Inclusão (ELASI) (OMOTE, 2005) foi aplicada de forma coletiva e monitorada, nas quatro escolas participantes. A ELASI é constituída por duas formas equivalentes (Forma A e Forma B). Cada forma é composta por 35 itens, sendo 30 para a mensuração de atitudes sociais frente à 
inclusão e outros cinco itens que compóem a escala de mentira. Os enunciados contemplam princípios político-filosóficos que norteiam a proposta da inclusão de uma forma ampla, ou seja, a da inclusão social e enfatizam as açôes que visam implementar a prática da Educação Inclusiva. A escala de mentira é constituída por 5 itens, com as mesmas alternativas de resposta dos outros 30 enunciados que compóem a ELASI, e serve para verificar algum problema ocorrido na mensuração, resultante de desatenção, erro na execução das tarefas exigidas, tendência a falsear o resultado ou qualquer outra ocorrência que possa interferir na resposta. Nesta pesquisa foi utilizada apenas a Forma A da ELASI, já que náo houve necessidade de mensurar as atitudes sociais em dois momentos distintos. Foi calculado um escore de atitudes sociais em relação à inclusão para cada respondente por meio da soma dos pontos obtidos em 30 itens, excluídos os itens da escala de mentira.

Para investigar as atitudes sociais de todas as crianças participantes deste estudo, utilizou-se a Escala Infantil de Atitudes Sociais em Relação à Inclusão (BALEOTTI, 2006). Esta escala avalia as atitudes sociais de crianças em relação à inclusão de pessoas deficientes. A aplicação foi coletiva e monitorada, realizada nas quatro classes, perfazendo o total de 116 crianças. Para a análise dos dados obtidos por meio desse instrumento, foram calculados escores individuais de cada uma, que podem variar de -27 a 27.

Foi realizada também análise estatística dos resultados referentes às atitudes sociais. Os dados numérico-estatísticos foram apresentados por meio de medidas de variação (valores mínimo e máximo), de tendência central (mediana) e de dispersão (quartil 1 e quartil 3). Empregou-se a Prova de Kruskal-Wallis e a Prova de Dunn para comparar os escores das atitudes sociais dos diferentes grupos. Foi utilizado o nível crítico de significância de $1 \%(\mathrm{p}<0,01)$.

\section{Resultados}

Primeiramente são apresentados os dados sobre as atitudes sociais dos professores frente à inclusão. A escala de mentira evidenciou que dos 73 professores que responderam a Forma A da ELASI, 64 atingiram escore 0 , sete o escore 1 e dois o escore 2 , o que indica que a maioria dos professores compreendeu os enunciados propostos e indicou com fidelidade e seriedade o nível de concordância ou de discordância em relação à inclusão.

No que se refere à análise de dados dos 30 itens da ELASI que efetivamente mensuram as atitudes sociais em relaçáo à inclusão, são apresentadas a medida de variação, representada pelo menor e maior escore, a medida de tendência central, representada pela mediana, e a medida de dispersão, representada pelo quartil $1 \mathrm{e}$ quartil 3 (Tabela 1). 
Tabela 1 - Síntese da distribuição de escores obtidos na ELASI de educadores das quatro escolas participantes.

\begin{tabular}{lllll}
\hline & $\begin{array}{l}\text { Escola P1 } \\
(\mathrm{n}=22)\end{array}$ & $\begin{array}{l}\text { Escola P2 } \\
(\mathrm{n}=25)\end{array}$ & $\begin{array}{l}\text { Escola controle 1 } \\
(\mathrm{n}=12)\end{array}$ & $\begin{array}{l}\text { Escola controle 2 } \\
(\mathrm{n}=14)\end{array}$ \\
\hline Variaçáo (Mín. - & $86,0-149,0$ & $98,0-149,0$ & $77,0-150,0$ & $99,0-146,0$ \\
Máx.) & & & & \\
Mediana & 124,0 & 126,0 & 122,5 & 127,0 \\
Dispersáo (Q1 - Q3) & $110,2-135,7$ & 111,0 & $-106,00-137,7$ & $108,2-138,2$ \\
& & 138,5 & & \\
\hline
\end{tabular}

A análise da Tabela 1 sugere que os professores das escolas que atendem alunos com TEA apresentam escores de atitudes sociais em relação à inclusão muito próximos dos valores apresentados por professores das escolas sem experiência de inclusão. Para comparar os quatro grupos concomitantemente foi utilizada a prova de Kruskal-Wallis e verificou-se não haver diferença estatística significante $(\mathrm{p}=0,949)$ nos escores obtidos. Assim, os grupos de professores das escolas dos P1 e P2 foram estatisticamente semelhantes aos grupos dos professores das escolas sem experiência de inclusão com relação às atitudes sociais mensuradas.

As professoras dos P1 e P2 também responderam à ELASI, sendo interessante situá-las dentro do seu respectivo grupo de professores que atuam na mesma escola. A professora de P1 obteve escore 146,0 (entre Q3 = 135,7 e máximo $=149,0)$ e a professora de P2 obteve escore 138 (entre Q3 = 138,5 e máximo = 149,0). Portanto, ambas localizam-se no quarto superior na distribuição dos escores dos professores que lecionam na mesma escola.

A Tabela 1 mostra a súmula dos escores das atitudes sociais em relação à inclusão, obtidos nas quatro classes que participaram deste estudo. São apresentadas as medianas, as variaçôes e os quartis dos escores da Escala Infantil. Os resultados das duas classes dos participantes focais não incluem os escores obtidos por estes.

Tabela 2 - Síntese dos resultados da Escala Infantil de Atitudes Sociais em Relação à Inclusão.

\begin{tabular}{lllll}
\hline & $\begin{array}{l}\text { Colegas de classe } \\
\text { P1 }(\mathrm{n}=30)\end{array}$ & $\begin{array}{l}\text { Colegas de classe P2 } \\
(\mathrm{n}=21)\end{array}$ & $\begin{array}{l}\text { Escola controle } \\
1(\mathrm{n}=32)\end{array}$ & $\begin{array}{l}\text { Escola } \\
\text { controle } \\
(\mathrm{n}=31)\end{array}$ \\
\hline Variaçáo (Mín. - Máx.) & $-2,0-21,0$ & $-10,0-19,0$ & $-14,0-22,0$ & $-21,0-19,0$ \\
Mediana & 12,0 & 1,0 & 2,5 & $-1,0$ \\
Dispersáo (Q1 - Q3) & $5,0-14,5$ & $-3,0-7,0$ & $-3,7-6,7$ & $-6,0-6,5$ \\
\hline
\end{tabular}

Prova de Kruskal-Wallis; Prova de Dunn

Verificou-se, por meio da prova de Kruskal-Wallis, que a diferença entre os grupos de alunos é altamente significante $(\mathrm{KW}=22,35 ; \mathrm{p}<0,0001)$. A prova de Dunn indica que a diferença reside na comparação entre os colegas de P1 e as crianças de cada uma das demais escolas, sendo que os colegas do P1 apresentam atitudes 
sociais mais favoráveis em relação à inclusão. Entre as outras três classes, não foi verificada nenhuma diferença significante. Os P1 e P2 também responderam à Escala Infantil de Atitudes Sociais em relação à Inclusão. O P1 obteve escore 4,0, localizando-se no quarto inferior na distribuição dos escores de seus colegas (entre mínimo = -2 e Q1 = 5). Já o P2 obteve escore -15, localizando-se abaixo do valor mínimo da sua classe $($ mínimo $=-10)$.

\section{Discussão}

A análise dos resultados nos quatro grupos de professores permitiu observar que não há diferenças na favorabilidade em relação à inclusão. Os professores das escolas dos alunos com transtornos do espectro do autismo (P1 e P2), foram estatisticamente semelhantes aos grupos dos professores das escolas sem experiência de inclusão para alunos com deficiências. Estes dados são corroborados por outros achados (CAMPBELL, 2006) que evidenciaram que apenas a experiência de contato não traz necessariamente mudança de atitudes sociais frente a crianças com transtornos globais do desenvolvimento. No Brasil, os resultados encontrados por Delgado e Omote (2010) sugerem que, para professores de Ensino Fundamental, a experiência com alunos surdos também não afetou a aceitação do processo de inclusão.

Evidentemente, as atitudes de professores em relação à inclusão são influenciadas também por uma miríade de fatores, tais como as políticas de inclusão, os recursos disponíveis na escola, o nível de apoio para atender às necessidades das crianças com deficiência (HSIEN, 2007; ROMI; LEYSER, 2006), variáveis pessoais do professor como o gênero e a idade (OMOTE, et al., 2005), o tempo de experiência docente (OMOTE et al., 2005; AVRAMIDIS; NORWICH, 2002), e a formação profissional (AVRAMIDIS; KALYVA, 2007; HSIEN, 2007; ROMI; LEYSER, 2006; OMOTE, et al., 2005). Para Campbell (2006), a melhora das atitudes sociais frente a crianças com transtornos globais do desenvolvimento depende de um ambiente que promova interaçóes adequadamente positivas e que favoreça oportunidades para as pessoas desenvolverem atitudes sociais mais adequadas.

As professoras dos P1 e P2 mostraram atitudes sociais mais favoráveis em relação à inclusão, quando comparadas às colegas que lecionavam nas mesmas escolas, pois seus escores se localizavam no quarto superior da distribuição dos escores dos respectivos grupos. É possível que o contato direto e cotidiano das professoras com os alunos com a transtornos do espectro do autismo tenha influenciado o fato de suas professoras terem apresentado maior favorabilidade das atitudes sociais frente à inclusão. Tais dados concordam com os resultados de outros estudos sobre a interferência positiva exercida pela experiência de contato com crianças com deficiências (OMOTE; PEREIRA, 2011; AVRAMIDIS; KALYVA, 2007; ROMI; LEYSER, 2006; AVRAMIDIS; NORWICH, 2002).

Por outro lado, em alguns casos, tal experiência de contato poderia originar atitudes pouco favoráveis, decorrentes, por exemplo, de situaçóes de estresse vivenciadas (AVRAMIDIS; NORWICH, 2002). Relatou-se que professores de crianças com transtornos globais do desenvolvimento podem apresentar ansiedade, depressão 
(GADOW et al., 2004) e estresse (PROBST; LEPPERT, 2008), muitas vezes relacionados às características típicas desses quadros, como comportamentos estereotipados (CONROY, et al., 2005), autoagressividade (FARMER; AMAN, 2010) e maior envolvimento em atividades solitárias e exploratórias (WILLIAMS, 2003). Além disso, professores frequentemente referiram que se sentem mal preparados para atender às necessidades comportamentais e de aprendizagem de alunos com transtornos globais do desenvolvimento (MARKS, et al., 2003); queixam-se também da falta de apoio e de aconselhamento para a prática (HELPS, et al., 1999).

No que tange às atitudes sociais das crianças, a partir das quatro escolas analisadas, pode-se verificar que os colegas de classe do P1 apresentaram atitudes sociais mais favoráveis em relação à inclusão, quando comparados aos colegas do P2 e às crianças do grupo controle. O P1 apresentava quadro de comprometimento menos severo que P2. Esta pode ser uma das razóes pelas quais a experiência de contato tenha contribuído para que houvesse mudança positiva nas atitudes sociais das crianças da classe de P1. O estudo de Baleotti (2006) também traz indicativos nessa direçâo.

A favorabilidade das atitudes sociais infantis pode estar associada tanto ao contato anterior das crianças com colegas com transtornos de desenvolvimento (CHAMBRES, et al., 2008; CAMPBELL, 2006) quanto ao tipo e ao grau de severidade das dificuldades da criança (BALEOTTI, 2006; CAMPBELL, 2006). Na presente pesquisa, os problemas de comportamento associados à agressividade demonstrados pelo P2, conforme observado nas avaliaçóes de linguagem e de comportamento, podem ter determinado contatos menos positivos com seus colegas. Tal achado é coerente com outros estudos (CHAMBRES, et al., 2008; BALEOTTI, 2006) que mencionaram também como um importante elemento envolvido na favorabilidade das atitudes sociais o fato de os contatos sociais terem sido ou náo positivos.

Segundo Chambres et al. (2008) e Campbell (2006), especialmente a discrepância entre distúrbios de comportamento expressos por crianças com transtornos globais do desenvolvimento e a aparência física normal pode implicar atitudes sociais negativas, pelo fato de não haver explicação visível e clara para a ocorrência de comportamentos incomuns. Omote (2004) apontou que a combinaçấo de determinadas qualidades que as pessoas julgam como sendo uma incongruência causa especial estranheza e pode mobilizá-las a buscar meios de redução ou eliminação dessa dissonância, sentida como cognitivamente desconfortável. Conforme o autor, algumas diferenças chamam atenção especial, seja por temor ou por desconfiança. Porém não é o atributo ou comportamento que tem inerente esse caráter, mas depende de como são interpretados pelo grupo social. Assim, um mesmo comportamento pode ser considerado desviante ou não, dependendo de quem o realiza, quem o julga e qual o contexto em que o julgamento ocorre.

Nesse sentido, Chambres et al. (2008) relataram que os mesmos observadores podem ser mais tolerantes ao mesmo tipo de comportamento se souberem que a criança apresenta um transtorno global do desenvolvimento. Se o comportamento dessa criança não é alterado ou é positivo, as avaliaçôes de pessoas informadas sobre seu diagnóstico podem ainda ser altamente favoráveis. 
Klin (2006) apontou que muitas crianças com transtornos do espectro do autismo inseridas em escolas comuns são especialmente vulneráveis a serem julgadas como excêntricas e a serem alvos de chacotas. Mas, segundo o autor, nos casos adequadamente assistidos, foram observados prognósticos positivos, com bons resultados para a aprendizagem escolar e inclusive com implicaçóes para a obtenção de emprego, independência e constituição de uma família na vida adulta. Os dados aqui levantados, além de permitirem examinar as problemáticas das relaçóes vivenciadas em ambiente escolar e a dificuldade para promover a educação escolar efetivamente inclusiva, podem indicar e fundamentar alternativas para o desenvolvimento e aplicação de intervençóes, que visem o desenvolvimento de potencialidades das crianças com e sem deficiências.

Omote et al. (2005) sugerem que podem ser integradas à matriz curricular dos cursos de formação de professores, uma breve disciplina planejada prioritariamente para a construção de atitudes sociais genuinamente favoráveis à inclusão. Além disso, a inserção, em diferentes disciplinas, de tópicos que devem ser tratados em diversas áreas de conhecimento.

Os resultados da presente pesquisa chamam atenção também para a capacitação de professores em serviço, uma vez que a condição de ter na sua classe aluno com TEA não afetou absolutamente as atitudes sociais de professores de P1 e P2. Essa condição poderia representar uma oportunidade privilegiada para que ocorresse revisão de crenças, valores e práticas por parte desses professores, se devidamente assessorados. Além disso, os próprios alunos, colegas de P2, não apresentaram atitudes sociais diferentes das de seus pares de escolas sem inclusão, e portanto, nenhum contato com algum aluno deficiente. Talvez caiba aos professores a responsabilidade pela construçáo de ambiente social acolhedor na sua classe. Para tanto, naturalmente o próprio professor precisa de atitudes sociais genuinamente favoráveis à inclusão.

Vieira (2014) adotou um delineamento que se mostrou efetivo para modificar as atitudes sociais de professores e respectivos alunos simultaneamente. Foi realizada uma intervenção que capacitou professores de uma escola do primeiro ciclo do ensino fundamental para aplicarem a seus alunos um programa informativo sobre a temática das deficiências e da inclusão voltado ao público infantil, que utilizava estratégias pedagógicas e lúdicas variadas. Os resultados indicaram que tanto professores quanto alunos modificaram suas atitudes sociais em relação à inclusão após passarem pela intervenção, tornando-as mais positivas do que antes e do que as do grupo controle. Neste formato de intervenção, percebe-se o potencial de ampliação da abrangência dos efeitos pretendidos, uma vez que os docentes poderão utilizar desta intervenção com outros alunos, em anos posteriores.

O TEA constitui em um quadro ainda pouco conhecido dos professores no Brasil, embora sua presença na escola venha aumentando progressivamente. Em razão de uma série de características já apontadas, destacando-se a ocorrência de comportamentos antissociais e a discrepância entre estes e a aparência das crianças com TEA, é particularmente importante direcionar a atenção para o meio social delas na escola. 
O trabalho de Vieira (2014) traz um bom exemplo de, em serviço, os professores serem capacitados para lidarem com questóes que envolvem conhecimentos e atitudes sociais favoráveis à inclusão. As variáveis pessoais tanto de professores quanto dos alunos podem ser convenientemente gerenciadas para a construçáo de um ambiente acolhedor em cada sala de aula, independentemente de haver ou não algum aluno com deficiência.

A pesquisa ora relatada deixou evidente que a mera oportunidade de convívio pode náo modificar as atitudes sociais nem dos professores nem de colegas de classe. Dada a relevância do ambiente acolhedor para a escolarização bem sucedida de todos os alunos, não se pode deixar eventuais mudanças nas atitudes sociais à mercê da ocorrência de eventos fortuitos. Parcela da responsabilidade pela construçáo desse ambiente acolhedor é do professor. Além de tornar as atitudes sociais dos professores e alunos favoráveis à inclusão, o trabalho de Vieira (2014) pode indicar que as questóes interacionais na sala de aula fazem parte do objeto de trabalho do professor.

\section{Referências}

ALAHBABI, A. K. Special And General Education Teachers' Attitudes Toward The Inclusion Of Students With Special Needs In General Education Classes In The United Arab Emirates (UAE), International Journal of Special Education, v. 24, n. 2, p.42-54, 2009.

ASSUMPÇÃO, JR.F.B., BAPTISTA F., GONÇALVES J.D.M., CUCCOLICHIO S., AMORIM L.C.D., REGO F., et al. Escala de avaliaçáo de traços autísticos (ATA): segundo estudo de validade. Med Reabil 2008; $27(2): 41-4$.

AVRAMIDIS, E.; KALYVA, E. The influence of teaching experience and professional development on Greek teachers' attitudes towards inclusion. European Journal of Special Needs Education, v.22, n.4, p.367-389, 2007.

AVRAMIDIS, E.; NORWICH, B. Teachers attitudes towards integration/inclusiun: a review of the literature. European Journal of Special Needs Education, v. 17, n. 2, p 129-147, 2002.

BALEOTTI, L.R, Um estudo do ambiente educacional inclusivo: descrição das atitudes sociais em relação à inclusão e das relações interpessoais. Tese (Doutorado em Educação) Universidade Estadual Paulista, Marília, 2006.

BEFI-LOPES, D.M. Vocabulário. In: Andrade CRF, Befi-Lopes DM, Fernandes FDM, Wertzner HF. ABFW: teste de linguagem infantil nas áreas de fonologia, vocabulário, fluência e pragmática. Pró-Fono: Carapicuiba; 2004.

CAMPBELL, M. J. Brief Report: Reliability and Validity of the Shared Activities Questionnaire as a Measure of Middle School Students' Attitudes toward Autism. Journal of Autism and Developmental Disorders. September 2008, Volume 38, Issue 8.

CAMPBELL, J.M.; MARINO, C.A. Brief report: sociometric status and behavioral characteristics of peer nominated buddies for a child with autism. J Autism Dev Disord. 2009; 39(9):1359-63.

CAMPBELL J.M. Changing Children's Attitudes Toward Autism: A Process of Persuasive Communication. Dev Phys Disabil, v. 18, n. 3, p. 251-272, 2006.

CONROY, M. A.; ASMUS, J. M.; SELLERS J. A.; LADWIG, C. N. The Use of an Antecedent-Based Intervention to Decrease Stereotypic Behavior in a General Education Classroom: A Case Study. Focus Autism Other Dev Disabil, v. 20, n.4, p. 223-230, 2005.

CHAMBRES, P., AUXIETTE, C., VANSINGLE, C.; GIL, S. Adult Attitudes Toward Behaviors of a Six-year -old Boy with Autism. J Autism Dev Disord, v. 38, n. 1, p.1320-27, 2008.

DELGADO-PINHEIRO, E. M. C.; OMOTE, S. Conhecimentos de professores sobre perda auditiva e suas atitudes frente á inclusão. Rev. CEFAC. v.12. n.4, p.633-640, 2010.

FARMER, C.A.; AMAN, M.G. Psychometric properties of the Children's Scale of Hostility and Aggression: Reactive/Proactive (C-SHARP). Res Dev Disabil. v.31, n.1, p.270-80, 2010 
FERNANDES, F.D.M. Pragmática. In: ANDRADE, C.R.F, BEFI-LOPES, D.M., FERNANDES, F.D.M. \& WERTZNER, H.F. ABFW: teste de linguagem infantil nas áreas de fonologia, vocabulário, fluência e pragmática. Pró-Fono: Carapicuiba; 2004.

GADOW, K. D. et al. Psychiatric symptoms in preschool children with PDD and clinic and comparison samples. J Autism Dev Disord. v.34, n.4, p. 379-93, 2004.

HELPS S.; NEWSOM-DAVIS I.; AND CALLIAS M. Autism the teacher's view. Autism, v.3, n.3, p. 287-298, 1999.

HSIEN, M. L. W. Teacher Attitudes towards Preparation for Inclusion - In Support of a Unified Teacher Preparation Program. Post-Script: Postgraduate Journal of Education Research. v.8, n.1, p. 49-60, 2007.

KLIN, A. Autismo e síndrome de Asperger: uma visão geral. Revista Brasileira de Psiquiatria, 28(Suppl. 1), s3-s11, 2006.

MARKS, S. U., SHAWV-HEGWER, J., SCHRADER, C., LONGAKER, T., PETERS, I., POWERS, F.; LEVINE, M. Instructional management tips for teachers of students with autism spectrum disorder (ASD). Teach Except Child, v.35, n.4, p.50-55, 2003.

OMOTE, S.; PEREIRA, A.A.J. Atitudes Sociais de Professoras de um Município de Médio Porte do Paraná em Relação à Inclusão. Pesquisas e Práticas Psicossociais, v. 6, n. 1, São João Del-Rei, janeiro/julho 2011.

OMOTE, S., OLIVEIRA, A.A.S., BALEOTTI, L.R.; MARTINS, S.E.S.O. Mudança de atitudes sociais em relação à inclusão. Paidéia. 2005;15(32):387-98.

OMOTE, S. A construção de uma escala de atitudes sociais em relação à inclusão: notas preliminares. Revista Brasileira de Educaçáo Especial. v.11, n.1, p.33-47, 2005.

OMOTE, S. Estigma no tempo da inclusáo. Rev Bra Educ Espec. 2004;10(3):287-308.

PROBST, P.; LEPPERT, T. Brief report: outcomes of a teacher training program for autism spectrum disorders. J Autism Dev Disord.v. 38, n. 9, p.1791-6, 2008.

ROMI, S. \& LEYSER, Y. Exploring inclusion preservice training needs: a study of variables associated with attitudes and self-efficacy beliefs. European Journal of Special Needs Education. v.21, n, 1, p. 85-105, 2006.

SWAIM, K.F. \& MORGAN, S.B. Children's Attitudes and Behavioral Intentions Toward a Peer with Autistic Behaviors: Does a Brief Educational Intervention Have an Effect? J Autism Dev Disord, v. 31, n. 2, p. 195$205,2001$.

VIEIRA, C. M. Atitudes sociais em relaçáo à inclusáo: efeitos da capacitação de professores para ministrar programa informativo aos alunos. Tese (Doutorado em Educação) - Universidade Estadual Paulista, Faculdade de Filosofia e Ciências. Marilia, SP, 2014.

WILLIAMS, E. A Comparative Review of Early Forms of Object-Directed Play and Parent-Infant Play in Typical Infants and Young Children With Autism. Autism, v. 7, n. 4, p. 361-377, 2003.

\section{Agradecimentos}

Os autores agradecem o apoio financeiro da Fundação de Amparo à Pesquisa do Estado de São Paulo - FAPESP.

\section{Correspondência}

Maria Cláudia Brito - Universidade Estadual Paulista Júlio de Mesquita Filho, Faculdade de Filosofia e Ciências - Campus de Marília. Consultório Particular - Bauru/SP; Laboratório de Estudos das Alteraçóes da Linguagem Infantil, Departamento de Fonoaudiologia; Programa de Pós-Graduação em Educação. Avenida Hygino Muzzy, 737. CEP: 17525900. Marília, São Paulo, Brasil.

E-mail: contato@saberautismo.com.br

Recebido em 11 de julho de 2017

Aprovado em 11 de agosto de 2017

Revista Educação Especial | v. 30 | n. 59 | p. 657-668 | set./dez. 2017

Santa Maria

Disponível em: <https://periodicos.ufsm.br/educacaoespecial> 
